\title{
A Quantum Hydrodynamic Simulation of Strained Nanoscale VLSI Device
}

\author{
Shih-Ching Lo ${ }^{1}$ and Shao-Ming $\mathrm{Yu}^{2}$ \\ ${ }^{1}$ National Center for High-Performance Computing, Computational Engineering Division \\ No. 7, R\&D $6^{\text {th }}$ Rd., Science-Based Industrial Park, Hsinchu, 300, Taiwan \\ sclo@nche.org.tw \\ ${ }^{2}$ National Chiao-Tung University, Department of Computer and Information Science \\ No. 1001, Ta Hsueh Road, Hsinchu, 300, Taiwan \\ smyu.cis91g@nctu.edu.tw
}

\begin{abstract}
Strained silicon field effect transistor (FET) has been known for enhancing carrier mobility. The stained $\mathrm{Si}$ channel thickness, the $\mathrm{Si}_{1-\mathrm{x}} \mathrm{Ge}_{\mathrm{x}}$ composition fraction and the $\mathrm{Si}_{1-\mathrm{x}} \mathrm{Ge}_{\mathrm{x}}$ layer thickness are three crucial parameters for designing strained $\mathrm{Si} / \mathrm{SiGe}$ MOSFET. Mobility enhancement and device reliability may be unnecessarily conservative. In this paper, numerical investigation of drain current, gate leakage and threshold voltage for strained $\mathrm{Si} / \mathrm{SiGe}$ MOSFET are simulated under different device profiles. According to our results, the optimal combination of parameters are as follows: stained Si channel thickness is $7 \mathrm{~nm}$, Ge content is $20 \%$, and the $\mathrm{Si}_{1-\mathrm{x}} \mathrm{Ge}_{\mathrm{x}}$ layer thickness should be chosen between $20 \sim 50 \mathrm{~nm}$.
\end{abstract}

\section{Introduction}

The introduction of strained $\mathrm{Si}$ and $\mathrm{SiGe}$ in CMOS technology is a means of improving the performance of Metal-Oxide-Semiconductor Field Effect Transistors (MOSFETs) in the deep submicron era [1-2]. A general approach to introduce biaxial tensile strain is using a virtual substrate of SiGe [1-2]. The underlying SiGe layer serves as an anchor to constrain the lattice of the strained silicon on top. Therefore, the electron mobility, and hence nMOSFET drive current performance, is enhanced. The stained Si channel thickness $\left(T_{S i}\right)$, the $\mathrm{Si}_{1-\mathrm{x}} \mathrm{Ge}_{\mathrm{x}}$ composition fraction $(x)$ and the $\mathrm{Si}_{1-\mathrm{x}} \mathrm{Ge}_{\mathrm{x}}$ layer thickness $\left(T_{S G}\right)$ are three crucial parameters for designing strained Si/SiGe MOSFET. In this study, computer-aided design (CAD) approach is used to optimize the structure of strained $\mathrm{Si} / \mathrm{SiGe}$ device. Drain current, gate leakage and threshold voltage are simulated and discussed.

\section{Quantum Transport Models}

The density-gradient (DG) model is considered to couple with the classical transport models. The hydrodynamic model (HD) [3] provides a very good compromise of velocity overshoot and the impact ionization generation rates. For the sake of saving computing time, DD model is used while drain bias $\left(V_{D}\right)$ is low $(<0.1)$. For high-drain bias, hydrodynamic model is considered. The DD model is given as 


$$
\begin{aligned}
& \nabla \varepsilon \cdot \nabla \phi=-q\left(p-n+N_{D}-N_{A}\right), \\
& q \partial n / \partial t-\nabla \cdot \mathbf{J}_{\mathbf{n}}=-q R, \\
& q \partial p / \partial t+\nabla \cdot \mathbf{J}_{\mathbf{p}}=-q R,
\end{aligned}
$$

where $\mathbf{J}_{n}=-q n \mu_{n} \nabla \phi_{n}$ and $\mathbf{J}_{p}=-q p \mu_{p} \nabla \phi_{p}$ are the electron and hole current densities. $\phi_{n}=-\nabla \phi-\nabla n\left(k T / \mu_{n}\right)$ and $\phi_{p}=-\nabla \phi+\nabla p\left(k T / \mu_{n}\right) . R$ is the generation-recombination term. In the hydrodynamic model, the carrier temperatures $T_{n}$ and $T_{p}$ are not assumed to be equal to lattice temperature $T_{L}$, together with DD model, up to three additional equations can be solved to find the temperatures, which are

$$
\begin{aligned}
& \partial W_{n} / \partial t+\nabla \cdot \mathbf{S}_{\mathbf{n}}=\mathbf{J}_{\mathbf{n}} \cdot \nabla E_{C}+d W_{n} /\left.d t\right|_{\text {coll }}, \\
& \partial W_{p} / \partial t+\nabla \cdot \mathbf{S}_{\mathbf{p}}=\mathbf{J}_{\mathbf{p}} \cdot \nabla E_{V}+d W_{p} /\left.d t\right|_{\text {coll }}, \\
& \partial W_{L} / \partial t+\nabla \cdot \mathbf{S}_{\mathbf{L}}=d W_{L} /\left.d t\right|_{\text {coll }},
\end{aligned}
$$

where $\mathbf{J}_{n}=\mu_{n}\left(n \nabla E_{C}+k_{B} T_{n} \nabla n+f_{n}^{t d} k_{B} n \nabla T_{n}-1.5 n k_{B} T_{n} \nabla \ln m_{e}\right)$ and $\mathbf{J}_{p}=\mu_{p}\left(p \nabla E_{V}-k_{B} T_{p} \nabla p-f_{p}^{t d} k_{B} p \nabla T_{p}-\right.$ $\left.1.5 p k_{B} T_{p} \nabla \ln m_{h}\right)$ are current densities. $\mathbf{S}_{\mathbf{L}}, \mathbf{S}_{\mathbf{n}}$ and $\mathbf{S}_{\mathbf{p}}$ are energy fluxes and $d W_{n} /\left.d t\right|_{\text {coll }}$, $d W_{p} /\left.d t\right|_{\text {coll }}$ and $d W_{L} /\left.d t\right|_{\text {coll }}$ are the collision terms.

According to DG method, an additional potential $\Lambda$ is introduced into the classical density formula, which reads: $n=N_{C} \exp \left(\left(E_{F}-E_{C}-\Lambda\right) / k_{B} T\right)$. In this study, $\Lambda$ is given as $\Lambda=\hbar^{2} \beta\left[\nabla^{2}(\phi+\Lambda)-\beta(\nabla \phi+\nabla \Lambda)^{2} / 2\right\rfloor / 12 m$ [4]. The quantum transport systematic equations are discretized by the box discretization [5] and solved. After the drain current $\left(I_{D}\right)$ is obtained, the gate leakage $\left(I_{G}\right)$ and threshold voltage $\left(V_{T H}\right)$ are determined.

\section{Simulated Results and Discussion}

In the numerical studies, a $40 \mathrm{~nm}$ strained $\mathrm{Si} / \mathrm{SiGe}$ nMOSFETs is simulated. The device profile and simulated scenario are given in Fig. 1. According to previous experimental studies [1-2], the effects of interface trap are also considered in the numerical simulation.

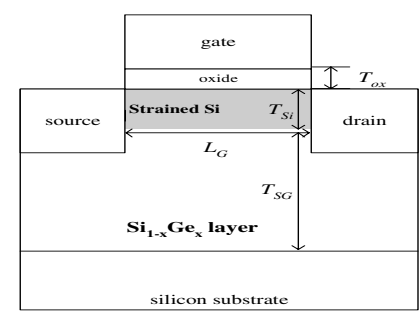

(a)

\begin{tabular}{ll}
\hline Applied bias: & \\
$V_{D}=1.25 \mathrm{~V}$, & $V_{G}=1.25 \mathrm{~V}$, \\
\hline Control variables: & $L_{G}=40 \mathrm{~nm}$, \\
$T_{O X}=1.5 \mathrm{~nm}$, & $N_{S D}=1 \times 10^{20} \mathrm{~cm}^{-3}$, \\
$N_{A}=1 \times 10^{18} \mathrm{~cm}^{-3}, \quad$ & \\
\hline simulated parameters: \\
$x=10 \%, 20 \%, 30 \%$ \\
$T_{S i}=5,7,9,11,15,20 \mathrm{~nm}$ \\
$T_{S G}=10,20,50,100,500 \mathrm{~nm}$ \\
\hline
\end{tabular}

(b)

Fig. 1. (a) Simulated strained $\mathrm{Si} / \mathrm{SiGe}$ nMOSFET and (b) scenario of simulation 
Figures 2 and 3 illustrate part of the results to show the dependence of $I_{D}$ and $I_{G}$ on $T_{S G}, T_{S i}$ and $x$, respectively. Fig. 2(a) demonstrates increasing device performance with increasing Ge content in the SiGe layer due to higher strain in the Si channel. However, a higher Ge content also induced higher gate leakage, which is caused by mismatch of lattice and diffusion of Ge. $I_{G}$ is shown in Fig. 3(a). Fig. 2(b) and Fig. 3(b) illustrate $I_{D}$ and $I_{G}$ under different $T_{S i}$ with $x=20 \%$ and $T_{S G}=20 \mathrm{~nm}$. A thinner $T_{S i}$ performs a larger $I_{D}$. The reason is that a thin strained Si channel can prevent the stress relaxation and present a better performance. The lattice mismatch proportionally decreases with increasing $T_{S i}$. In another word, the relaxation of tensile stress in the strained Si channel could be suppressed by decreasing $T_{S i}$, i.e., the mobility enhancement would be larger in the thin $T_{S i}$ device than in the thick $T_{S i}$ device. The observation in the nanoscale device is different to long-channel devices. Unfortunately, a thin $T_{S i}$ may induce large interface trap caused by Ge diffusion. In this study, $I_{D}$ and $I_{G}$ of $T_{S i}=5 \mathrm{~nm}$ is the largest. $I_{G}$ of $T_{S i}=7,9,11$ and 15 do not show much difference. Fig. 2(c) and 3(c) demonstrate $I_{D}$ and $I_{G}$ dependence on $T_{S G}$. A thicker $T_{S G}$

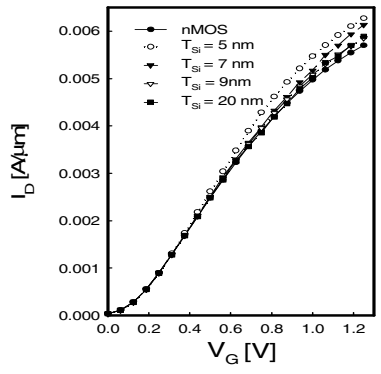

(a)

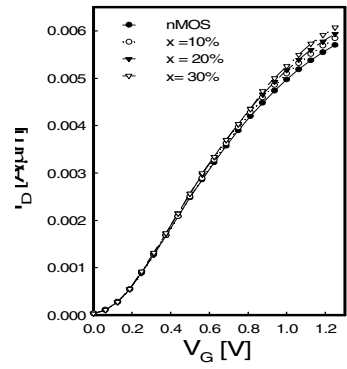

(b)

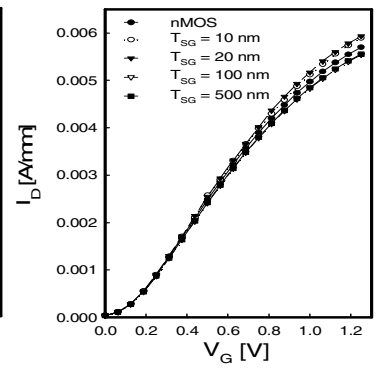

(c)

Fig. 2. Simulated $I_{D^{-}} V_{G}$ curves for (a) $T_{S G}=20 \mathrm{~nm}, x=20 \%$, (a) $T_{S G}=20 \mathrm{~nm}, T_{S i}=9 \mathrm{~nm}$ and (c) $T_{S i}=9 \mathrm{~nm}, x=20 \%$

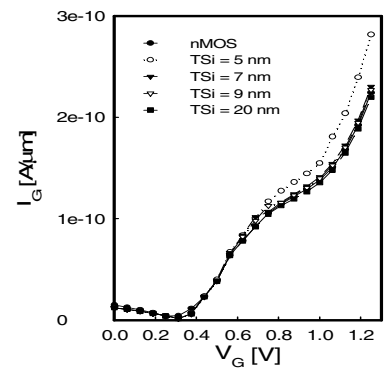

(a)

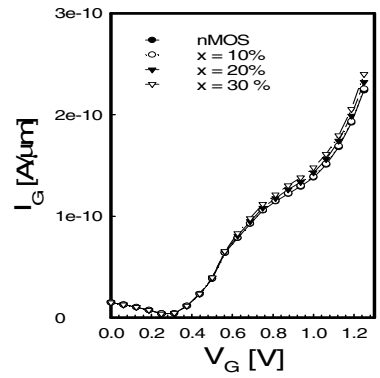

(b)

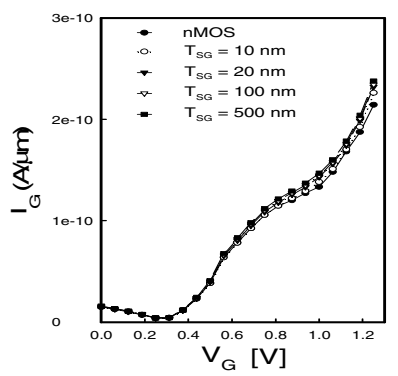

(c)

Fig. 3. Simulated $I_{G}-V_{G}$ curves for (a) $T_{S G}=20 \mathrm{~nm}, x=20 \%$, (a) $T_{S G}=20 \mathrm{~nm}, T_{S i}=9 \mathrm{~nm}$ and (c) $T_{S i}=9 \mathrm{~nm}, x=20 \%$ 
provides a larger stress, but a larger number of lattice mismatches is also induced. Moreover, a thicker $T_{S G}$ may increase Ge diffusion to MOS interface, which contributes to an increased interface state.

For further discussion, Fig. 4 illustrates the $V_{T H}$ shift $(\mathrm{V}), I_{D}$ enhancement (\%) and $\Delta I_{G} I_{G}(\%)$ of the whole simulation scenario. According to the figure, if $T_{S i} \geqq 14 \mathrm{~nm}$, $V_{T H}$ shift may be larger than $0.01 \mathrm{~V}$. As $T_{S i} \leqq 14 \mathrm{~nm}, V_{T H}$ shift is small enough to be neglected. From Fig. 4 (b), the largest $I_{D}$ enhancement is achieved by $T_{S i}=5 \mathrm{~nm}, T_{S G}$ $=20 \mathrm{~nm}$ and $\mathrm{Si}_{70 \%} \mathrm{Ge}_{30 \%}$. However, the $I_{G}$ induced by the interfacial state is too large to be accepted. $T_{S i}=5 \mathrm{~nm}$ presents a sudden increase of interfacial states. $\Delta I_{G} / I_{G}$ is given in Fig. 4(c). $T_{S i}=7 \mathrm{~nm}$ is chosen. Although $x=30 \%$ may have a better improvement of drain current, it also have a serious problem of Ge diffusion. Therefore, $x=20 \%$ is suggested. Since a thin SiGe layer cannot provide enough stress to improve drain current, the best case occurs between $T_{S G}=20 \sim 50 \mathrm{~nm}$.

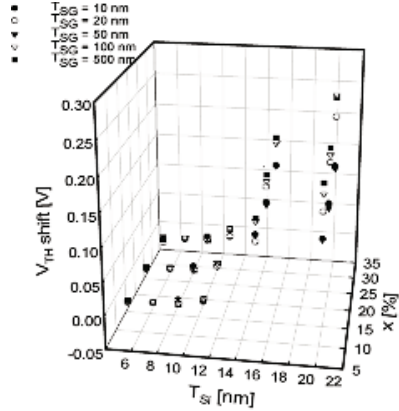

(a)

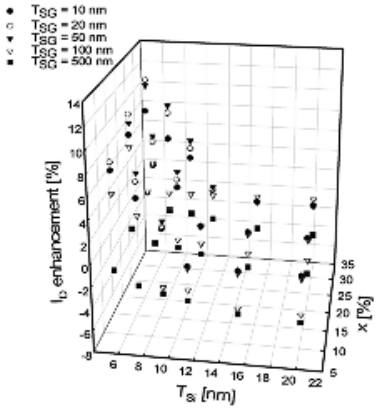

(b)

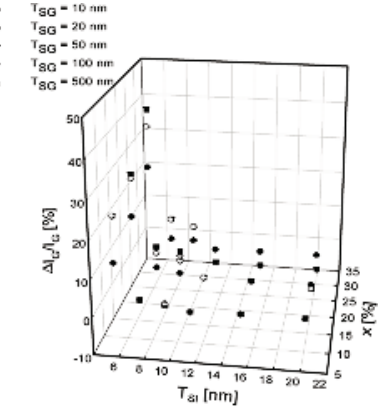

(c)

Fig. 4. (a) $V_{T H}$ shift (V), (b) $I_{D}$ enhancement (\%), and (c) $\Delta I_{G} / I_{G}(\%)$ for the simulated scenario

\section{Conclusions}

In this study, optimal profile of strained $\mathrm{Si} / \mathrm{SiGe}$ device is suggested by numerical simulation. Hydrodynamic model is employed and the effect of interface state is considered in the simulation. Considering the improvement of performance and reliability, we suggested that the optimal stained Si channel thickness is $7 \mathrm{~nm}, \mathrm{Ge}$ content is $20 \%$, and the $\mathrm{Si}_{1-\mathrm{x}} \mathrm{Ge}_{\mathrm{x}}$ layer thickness is between $20 \sim 50 \mathrm{~nm}$. The optimal design may obtain an $8 \sim 11 \%$ improvement of performance and maintain the same level of gate leakage for a $40 \mathrm{~nm}$ nMOSFET.

\section{Acknowledgement}

The authors would like to thank Professor Y. Li of Department of Communication Engineering, National Chiao Tung University, Taiwan for useful discussion. 


\section{References}

1. Kwa, K. S. K., Chattopadhyay, S., Olsen, S. H., Driscoll, L. S., and O;Neill, A. G.: Optimization of Channel Thickness in Strained Si/SiGe MOSFETs, in Proc. ESSDERC (2003) 501-504

2. Olsen, S. H., O;Neill, A. G., Driscoll, L. S., Chattopadhyay, S., Kwa, K. S. K., Waite, A. M., Tang, Y. T., Evans, A. G. R., and Zhang, J.:Optimization of Alloy Composition for High-Performance Strained-Si-SiGe N-Channel MOSFETs, IEEE Trans. Elec. Dev. 51 (2004) 1156-1163

3. Bløtekjær, K.: Transport Equations for Electrons in Two-Valley Semiconductors, IEEE Trans. Elec. Dev. ED-17 (1970) 38-47

4. Wettstein, A., Schenk, A. and Fichtner, W.: Quantum Device-Simulation with the DensityGradient Model on Unstructured Grids, IEEE Trans. Electron Devices 48 (2001) 279-284

5. Bürgler, J. F., Bank, R. E., Fichtner, W., and R. K. Smith,: A New Discretization Scheme for the Semiconductor Current Continuity Equations, IEEE Trans. CAD 8 (1989) 479-489. 